\title{
Comparison of Theoretical and Practical Performances with 802.11n and 802.11ac Wireless Networking
}

\author{
David Newell, Philip Davies, Russell Wade, Perry deCaux \\ Faculty of Science \& Technology \\ Bournemouth University \\ Dorset, UK \\ dnewell@bournemouth.ac.uk, daviesp@bournemouth.ac.uk, \\ rwade@bournemouth.ac.uk,pdecaux@bournemouth.ac.uk
}

\author{
Mak Shama \\ Birmingham City University \\ School of Computing \& Digital Technology \\ Birmingham, UK \\ mak.sharma@bcu.ac.uk
}

\begin{abstract}
This work explores the theoretical and practical performances of the two most recent IEEE standards, 802.11n and 802.11ac. Experiments were conducted to measure data rates to characterize performance effects of distance and interference between different channels. We conclude that the majority of test cases show 802.11ac achieved higher data rates than its predecessor, as expected. However, performance of 802.11ac decreased at a significantly faster rate with increasing distance from client to AP when compared to the decreasing performance experienced with $802.11 n$. Furthermore, 802.11n consistently achieved real data rates much closer to the theoretical data rate than did 802.11ac.
\end{abstract}

Keywords- Wireless Networks, Network Monitoring, IEEE Standards, IEEE 802.11

\section{INTRODUCTION}

Transmission standards often state a theoretical data rate that is rarely achieved in practice.

The contemporary wireless standards, IEEE 802.11n and $802.11 \mathrm{ac}$, promised data rates of up to $600 \mathrm{Mbs}$ and $6933 \mathrm{Mbs}$ respectively. This paper explores these two IEEE standards to discover their practical performance in an office-like environment. The theoretical capabilities and features of 802.11n and 802.11ac are also reviewed, and the data rates are tested using the lowest, mid and highest channel to compare the two standards' theoretical and practical performances.

Chen and Suzuki [1], Bejarano and Knightly [2], and Dianu et al. [3] have explored 802.11 amendment performances individually. The typical throughput of IEEE $802.11 \mathrm{a} / \mathrm{g}$ is $25 \mathrm{Mb} / \mathrm{s}$ with a physical-layer (PHY) data rate of $54 \mathrm{Mb} / \mathrm{s}$. The IEEE 802.11n standard was the first IEEE 802.11 amendment to introduce Multiple-Input-Multiple-Output (MIMO) by implementing spatial diversity. The 802.11n standard aimed to achieve at least four times that of $802.11 \mathrm{a} / \mathrm{g}$ with a capability of at least $100 \mathrm{Mb} / \mathrm{s}$ throughputs at the MAC layer.

\section{A. Protocols and Features $802.11 n$}

Innovative features which have been introduced with the new standards have included; Reduced Interframe Space (RIFS), Frame Aggregation, Transmit Beamforming (TxBF),
Space Time Block Codes (STBC), Low Density Parity Check (LDPC), Modulation and Coding Schemes (MCS) index and Spatial Streams.

Reduced Interframe Space (RIFS) was a new feature to improve efficiency and performance. Kassner [4] explains that RIFS reduces the amount of wasted time required between Orthogonal frequency-division multiplexing (OFDM) transmissions. This feature is only available in deployments, where the R.F. environment is free from legacy devices.

Frame aggregation was implemented to the MAC layer as the primary method for increasing efficiency and providing a MAC layer throughput of at least $100 \mathrm{Mb} / \mathrm{s}$. Frame aggregation is the method of increasing the data portion's length within the frane in order to increase overall efficiency.

Transmit Beamforming (TxBF) is another new feature popularly employed in $802.11 n$. TxBF can provide a better signal, greater Signal to Noise Ratio (SNR) and a higher throughput by concentrating the Access Point (AP) signal to a client's location.

STBC are orthogonal codes which can achieve full transmission diversity depending on the number of specified antennas [5]. STBC is a PHY layer implementation which improves link performance over MIMO with basic spatialdivision multiplexing (SDM) by achieving the maximum diversity order for the number of MIMO streams. SDM transmits independent data streams via multiple transmit antennas to increase throughput. Whereas, STBC improves performance by utilising spatial transmit diversity at a given bit rate [6].

Low-Density Parity-Check (LDPC) is a forward error correcting code and an optional PHY specification which contributes to better performance. According to Gast[7], if LDPC is enabled, it can provide an additional antenna gain of $1-2 \mathrm{~dB}$ and can also increase data rates.

The MCS index was created with the $802.11 \mathrm{n}$ amendment to determine theoretical data rates of $802.11 \mathrm{n}$ devices. The standard introduced high-throughput orthogonal frequency division multiplexing (HT-OFDM) because of the use of 
additional parameters such as spatial streams, channel size, coding method, modulation technique, and guard interval. Prior to $802.11 n$, standards which used OFDM had defined data rates between $6-54 \mathrm{Mb} / \mathrm{s}$ depending on the type of modulation and coding techniques used [8].

MIMO implementation introduced the term "spatial streams". The amendment is capable of achieving up to $4 \times 4$ spatial streams which provides a theoretical throughput of $600 \mathrm{Mb} / \mathrm{s}$. As the infrastructure is limited to $2 \times 2$ spatial streams at the client side, theoretically, it should be able to achieve throughput of $300 \mathrm{Mb} / \mathrm{s}$ in relation to its MCS index.

\section{B. Modulation Types}

There are two modulation types using $20 \mathrm{MHz}$ and $40 \mathrm{MHz}$ channels. Unlike previous 802.11 amendments, where only an available channel width of $20 \mathrm{MHz}$ is attainable, the.$n$ amendment introduced a larger channel width of $40 \mathrm{MHz}$. Two $20 \mathrm{MHz}$ channels are combined in order to provide the $40 \mathrm{MHz}$ channel width, potentially doubling the data rate. Increasing the bandwidth has a relatively low cost and as a result, has become an important feature of the 802.11n standard. Most 802.11n devices now support $40 \mathrm{MHz}$ bandwidth channels, but it is optional due to interoperability of $802.11 \mathrm{a} / \mathrm{b} / \mathrm{g}$ legacy devices which use $20 \mathrm{MHz}$ channels.

Table 1: Capabilities of 802.11n hardware and 802.11n IEEE specification (amended from Cisco 2015)

\begin{tabular}{l|l|l} 
& $\mathbf{8 0 2 . 1 1 n}$ & $\begin{array}{l}\mathbf{8 0 2 . 1 1 n} \\
\text { IEEE Specification }\end{array}$ \\
\hline Frequency Band: & $\begin{array}{l}\text { Dual Band } \\
2.4 \& 5 \mathrm{GHz}\end{array}$ & $\begin{array}{l}\text { Dual Band } \\
2.4 \& 5 \mathrm{GHz}\end{array}$ \\
\hline No. of Spatial Streams: & $3 \times 3$ & $4 \times 4$ \\
\hline Channel Width: & $20 / 40 \mathrm{MHz}$ & $20 / 40 \mathrm{MHz}$ \\
\hline Max Modulation Scheme: & $64-\mathrm{QAM}$ & $64-\mathrm{QAM}$ \\
\hline Max PHY Rate: & $450 \mathrm{Mb} / \mathrm{s}$ & $600 \mathrm{Mb} / \mathrm{s}$ \\
\hline Max MAC Throughput: & $293 \mathrm{Mb} / \mathrm{s}$ & $390 \mathrm{Mb} / \mathrm{s}$ \\
\hline Beamforming Support: & Vendor Specific & Vendor Specific \\
\hline MIMO Support: & Yes(SU-MIMO) & Yes (SU-MIMO) \\
\hline
\end{tabular}

\section{Technical Specification of 802.11ac}

The majority of the 802.11ac amendments propose significant efficiency enhancements to those introduced in 802.11n. An improvement to the MAC layer supports new physical layer features which provide considerably higher throughput and data rates. The increase in frame sizes within the amendment has contributed to increasing data rates and throughput.

Although RIFS reduces the amount of time between frame transmissions in $802.11 \mathrm{n}$, it is more efficient to aggregate frames instead. RIFS transmits two frames separately resulting in two full headers and Physical Layer Convergence Protocol (PLCP) frames being transmitted. In 802.11ac, a single Aggregated MAC Protocol Data Unit (A-MPDU) transmits two frames at once, increasing efficiency.

Frame Size and Aggregation Frame size has had several improvements in 802.11ac. Frame sizes have increased and each frame is now transmitted individually as an A-MPDU, even if it only contains a single frame. It may seem inefficient to transmit A-MPDU which only has a single frame. However, rather than sending a large number of bytes in a PLCP header which is then transmitted at the lowest possible data rate, 802.11ac moves the length indication to the MPDU which is then transmitted as part of the high data rate payload [7]. Ultimately, the most significant enhancement to frame size is Physical Layer Service Data Unit (PSDU) PLCP payload size which was increased from 0.06 MB to 4.45 MB.

The available spectrum is used by 802.11ac more efficiently by determining channel bandwidth on a frame-byframe basis. Primary and secondary channels are introduced which help divide airtime between channels so that the same frequency space can be used by multiple networks. Each channel bandwidth, such as $20 \mathrm{MHz}, 40 \mathrm{MHz}, 80 \mathrm{MHz}$, and $160 \mathrm{MHz}$ has one primary channel which transmits data at its native bandwidth. The evolution of wider channels in 802.11ac built upon $802.11 n$ which combines two channels together for transmission.

A single method for beamforming was introduced by 802.11ac. Null Data Packet (NDP) sounding reduces complexity by preventing the proprietary issues with $802.11 n$. Another major change to beamforming is multi-user MIMO (MU-MIMO). MU-MIMO was introduced in the second wave of hardware and is a technique which enables simultaneous transmission to multiple clients.

The data rate of 802.11ac is improved by a better modulation scheme. Previously, 64-QAM restricted potential data rates. Building on its predecessor, 802.11ac uses a better modulation type, with data rates four times higher that of 64QAM.

Table 2: Comparison of Wave 1 and 2 of 802.11ac hardware and the IEEE 802.11ac Specification (amended from Cisco 2015)

\begin{tabular}{l|l|l|l} 
& Wave 1 & Wave 2 & $\begin{array}{l}\text { 802.11ac } \\
\text { IEEE Specification }\end{array}$ \\
\hline Frequency Band: & $5 \mathrm{GHz}$ & $5 \mathrm{GHz}$ & $5 \mathrm{GHz}$ \\
\hline No. of Spatial Streams: & $3 \times 3$ & $3 \times 4$ & $8 \times 8$ \\
\hline Channel Width: & $20 / 40 / 80 \mathrm{MHz}$ & $\begin{array}{l}20 / 40 / 80 / 80-80 \\
/ 160 \mathrm{MHz}\end{array}$ & $\begin{array}{l}20 / 40 / 80 / 80-80 / 160 \\
\mathrm{MHz}\end{array}$ \\
\hline $\begin{array}{l}\text { Max Modulation } \\
\text { Scheme: }\end{array}$ & $256-\mathrm{QAM}$ & $256-\mathrm{QAM}$ & $\begin{array}{l}256-\mathrm{QAM} / 1024-\mathrm{QAM} \\
\text { (Quantenna 2015) }\end{array}$ \\
\hline Max PHY Rate: & $1.3 \mathrm{~Gb} / \mathrm{s}$ & $\begin{array}{l}2.5-3.47 \mathrm{~Gb} / \mathrm{s} \\
(\text { Cisco } 2015)\end{array}$ & $6.9 \mathrm{~Gb} / \mathrm{s}$ \\
\hline $\begin{array}{l}\text { Max MAC } \\
\text { Throughput: }\end{array}$ & $845 \mathrm{Mb} / \mathrm{s}$ & $\begin{array}{l}1.52-2.26 \mathrm{~Gb} / \mathrm{s} \\
\text { (Cisco 2015) }\end{array}$ & $4.49 \mathrm{~Gb} / \mathrm{s}$ \\
\hline Beamforming Support: & Vendor Specific & Yes & Yes \\
\hline $\begin{array}{l}\text { SU/MU MIMO } \\
\text { Support: }\end{array}$ & SU-MIMO & MU-MIMO & MU-MIMO \\
\hline
\end{tabular}

\section{EXPERIMENTAL DESIGN}

Dianu et al. [3] have measured 802.11ac and arrived at a similar expectation of practical performance and an indication of an array of variables to use for the experiment. Chen and Suzuki [1] have carried out experiments using MIMO in 802.11n over different channels and MCS numbers, similarly. Our experiment extended this work with additional variables that had not previously been considered. 


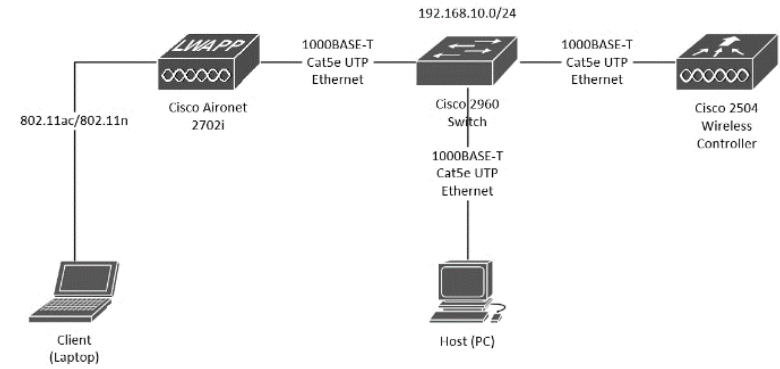

Fig 1: Physical network diagram of the proposed experimental infrastructure.

In summary, the tests record practical data rates of both standards at distances starting at zero metres up to twenty five metres in five metre increments. Tests for the lowest, mid and highest channel number were recorded and then formed into average data rate.

The frequency band used for the experiment was $5 \mathrm{GHz}$. Other variables under consideration are Received Signal Strength Indicator (RSSI), dBm, datagram size, and transport protocol. RSSI will provide an indication of signal strength at the client. Similarly, $\mathrm{dBm}$ is power received at the client from the transmitting antennas at the AP. The exact frequency of communication is recorded. Datagram size was recorded in bytes as the size of each datagram being generated as traffic. The transport protocol employs User Datagram protocol (UDP) for which WireShark [9] will be used to inspect network data. Analysis rows such as errors/packet loss, highest, lowest and average data rate will be recorded for evaluation purposes.

WirelessMon will be used to gather and record the data for each test case. The result is a radar representing signal strength from which graphs can be exported that contain data rates. There are several parameters within the graph which can be selected such as RSSI, $\mathrm{dBm}$, received data rate, sent data rate, and total data rate, all of which are of concern. Also present in graphs are fields for channel usage, wireless devices within the environment, number of antennas and transmit power. The WirelessMon software was the primary tool for gathering the data and AdapterWatch [10] will be used in parallel for verification.

\section{A. Experimental set up}

Changes, made to infrastructure configuration from the original design, are now described.

It was originally planned to record data rates at distances from five to thirty metres in five metre increments. However, the laboratory used allowed distances of up to fifteen meters only. This resulted in potentially measuring longer distances outside in open space. This is not a true representation of typical office environments. Instead, data rates were recorded up to twenty five metres by moving the client to the adjacent room, which better represents a typical office environment at distances from twenty to twenty five metres. Fig 2 shows the floor plan of the two rooms and the physical infrastructure.

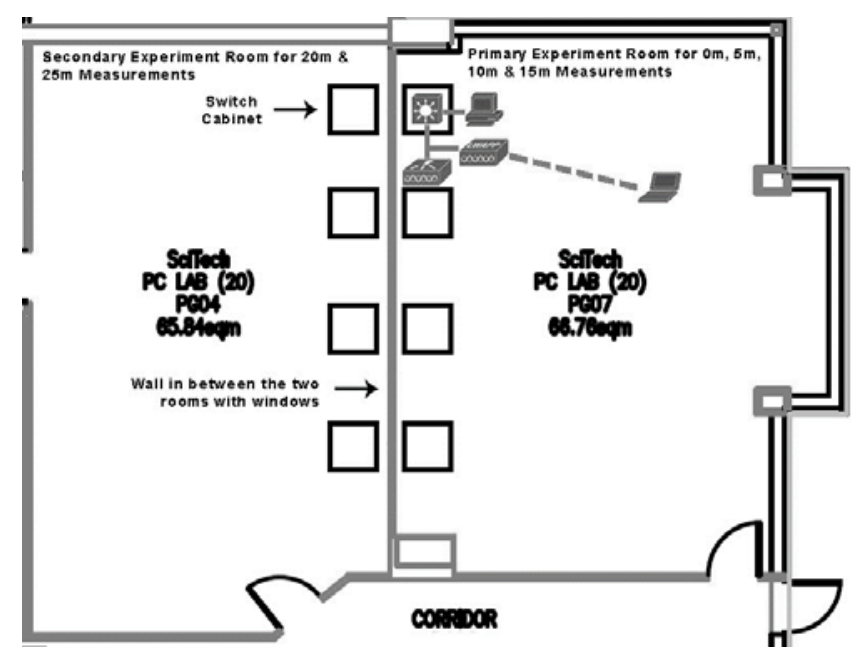

Fig 2: Floor plan of the laboratory infrastructure

\section{FINDINGS}

Three hundred and twenty results sets of the two standards practical performance were measured. Space only permits a summary of the results to be described.

\section{A. Average 802.11n Data Rate Over Each Channel}

Distances from twenty to twenty five metres where data rates were measured through a wall, data rate performance significantly drops over the mid and low channels. However, on the highest channel (136) data rates increased through the wall showing better performance at distances and through obstacles than its lower channels. Interestingly, the highest average data rate recorded $(164 \mathrm{Mb} / \mathrm{s})$ was at ten meters on channel 36 which had the highest number of APs (21) operating over the same channel, representing the highest potential interference.

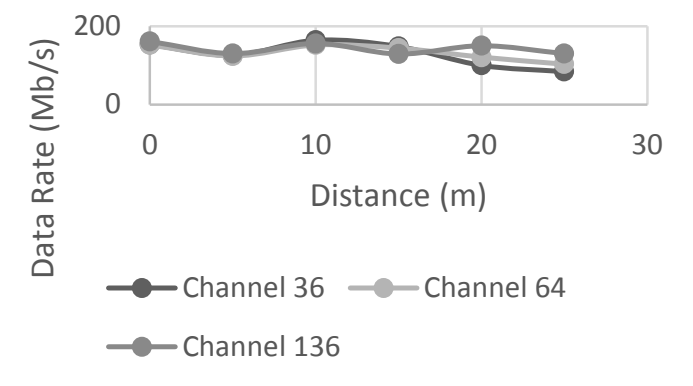

Fig 1: 802.11n average data rate over the lowest (Ch 36), mid (Ch 64), and highest (Ch 136) channels

\section{B. Highest 802.11n Data Rate Over Each Channel}

The average data rates recorded over the lowest, mid and highest channel in 802.11ac can be seen in Fig 4. The highest average data rate recorded was $282 \mathrm{Mb} / \mathrm{s}$ over the highest. Dissimilar from $802.11 \mathrm{n}$ average data rate, the mid channel over 802.11ac which had interference performed better at 
longer distances than the highest channel which had no interference. Average data rates recorded over channel 64 appear rather inconsistent compared to channels 36 and 112 which may be due to interfering APs transmitting at the same time.

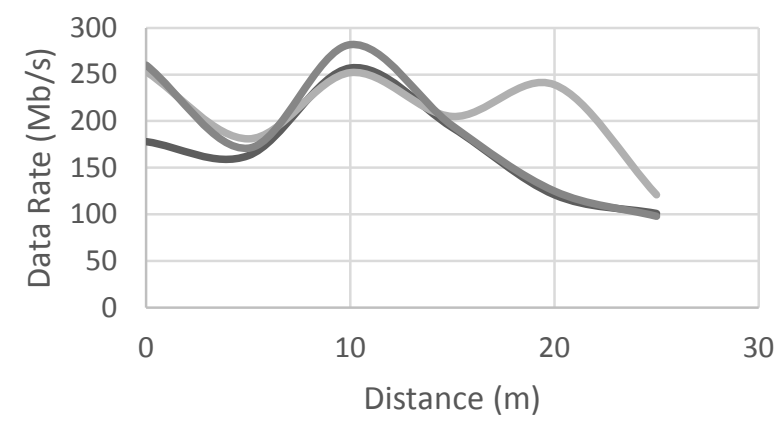

Channel $36 \longrightarrow$ Channel $64 \longrightarrow$ Channel 112

Fig 2: 802.11ac average data rate over the lowest (Ch 36), mid (Ch 64), and highest (Ch 112) channels

\section{C: Comparing 802.11n and 802.11ac Average Data Rates} Over the Lowest Channel

Fig 5 represents the comparison between $802.11 \mathrm{n}$ and 802.11ac average data rate over the mid channel. 802.11n has an extremely similar trend to its lower channel average which can be seen in Fig 7. However, 802.11ac's average varies considerably with spikes of data rates increasing and decreasing at each distance. This could be down to interfering APs transmitting simultaneously over the mid channel as 16 APs were present in the wireless environment. 802.11ac recorded the highest data rate at $253 \mathrm{Mb} / \mathrm{s}$ whereas $802.11 \mathrm{n}$ recorded $152 \mathrm{Mb} / \mathrm{s}$ at zero and ten meters. Both highest data rates recorded were similar to the lower channel results in Fig 7. A continuous pattern which emerges is the decrease in data rate with 802.11ac over longer distances where 802.11n was only $18 \mathrm{Mb} / \mathrm{s}$ less at the same distance.

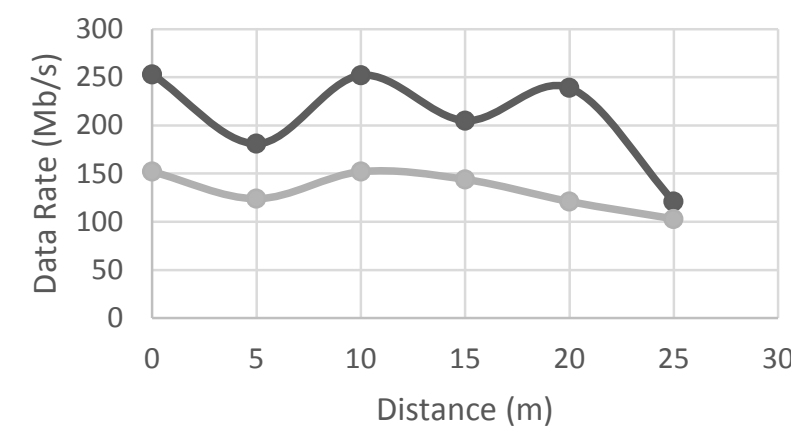

$-802.11 \mathrm{ac}(\mathrm{Ch} 64) \quad-802.11 \mathrm{n}(\mathrm{Ch} 64)$

Fig 3: A comparison of the average data rates recorded at the mid channel over both $802.11 \mathrm{n}$ and $802.11 \mathrm{ac}$

\section{Comparing 802.11n and 802.11ac Average Data Rates Over the Highest Channel}

A graph containing the comparison between 802.11n and 802.11ac's average data rate over the highest channel can be seen in Fig 6. 802.11n's performance doesn't show the same consistency shown in Fig 5. Instead, the data rate appears to fluctuate regardless of the distance or obstacles within the environment and shows the highest data rates recorded at further distances shown in in Fig 5. 802.11ac maintains inconsistency in performance beginning with a spike until data rates eventually decrease below 802.11n's data rates. 802.11ac managed to achieve $282 \mathrm{Mb} / \mathrm{s}$ at ten meters whilst $802.11 \mathrm{n}$ achieved $161 \mathrm{Mb} / \mathrm{s}$ at zero meters. Non-interference over the highest channel may be the reason why 802.11 n managed to maintain data rates at the furthest distance and also why 802.11ac achieved its highest average.

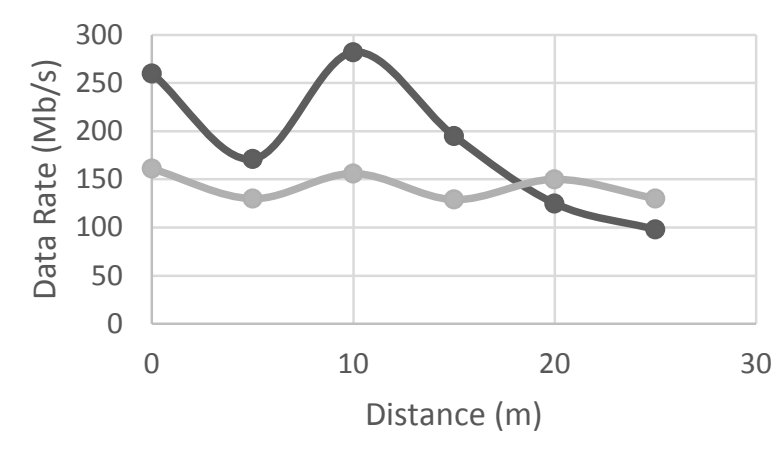

$-802.11 \mathrm{ac}$ (Ch 112) $\quad-802.11 \mathrm{n}$ (Ch 136)

Fig 4: A comparison of the average data rates recorded at the highest channel over both 802.11n and 802.11ac (Personal Collection)

\section{Comparing Combined 802.11n and 802.11ac Average Data Rates Over All Channels}

With the average data rate of the lowest, mid, and highest channels over 802.11n and 802.11ac compared and analysed, Fig 7 presents a combined average data rate over all three of the channels. IEEE 802.11n retained its performance with the highest data rates recorded at zero and ten metres which then steadily decreased as the distance increased. 802.11ac maintained its highest data rate at ten metres but then rapidly decreased to an average extremely close to $802.11 \mathrm{n}$. The highest total average data rate was $264 \mathrm{Mb} / \mathrm{s}$ for $802.11 \mathrm{ac}$ and $157 \mathrm{Mb} / \mathrm{s}$ for $802.11 \mathrm{n}$. The lowest however were nearly identical with $802.11 \mathrm{ac}$ recorded at $107 \mathrm{Mb} / \mathrm{s}$ and $802.11 \mathrm{n}$ at $106 \mathrm{Mb} / \mathrm{s}$. This leads onto the conclusion that 802.11ac appears rather volatile to its surroundings with a huge impact on performance over further distances compared to 802.11n. 


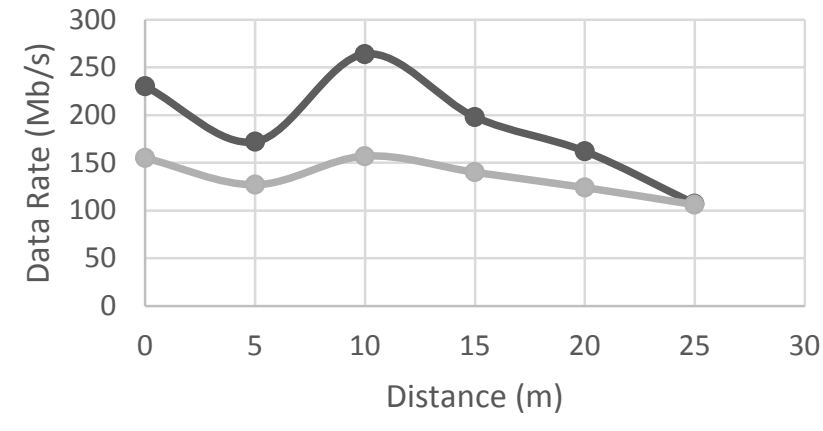

$-802.11 \mathrm{ac}-\mathrm{C}-\mathrm{C02.11n}$

Fig 5: A comparison of the average data rates recorded over the lowest, mid, and highest channels combined for both 802.11n and 802.11ac (Personal Collection)

\section{E. Highest 802.11n and 802.11ac Data Rate Over All Channels For Each Distance}

Fig 8 shows the comparison of 802.11n and 802.11ac's practical performance against their corresponding theoretical performance. Results in Fig 8 respectably support previous analysis within this chapter with evidence showing $802.11 n$ 's consistency over 802.11ac. 802.11n achieved on average $44.9 \%$ of its theoretical performance as opposed to 802.11ac's practical average performance of $21.7 \%$ of its theoretical performance. This trend continued for the highest data rates recorded with $802.11 \mathrm{n}$ achieving $84.4 \%$ of its theoretical performance compared to 802.11ac's 47\%. 802.11n also accomplished better performance with the lowest data rates recorded at $8.8 \%$ whereas $802.11 a c$ provided an average of just $2.6 \%$.

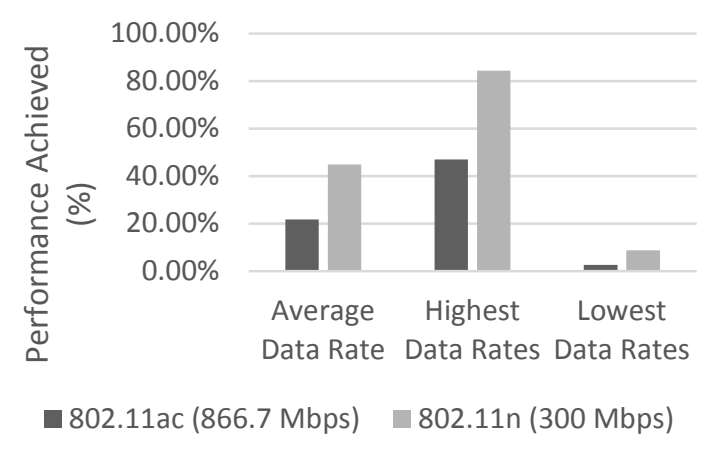

Fig 6: Comparison of $802.11 \mathrm{n}$ and $802.11 \mathrm{ac}$ practical performance against their theoretical performance.

\section{SUMMARY}

In terms of performance, 802.11n outperformed 802.11ac in relation to theoretical speeds. $802.11 n$ was also the closest to achieving its theoretical speed with $288 \mathrm{Mb} / \mathrm{s}$ whereas $802.11 \mathrm{ac}$ was far from its theoretical speed of $866.7 \mathrm{Mb} / \mathrm{s}$ with $510 \mathrm{Mb} / \mathrm{s}$. However at closer distances, higher practical speeds were attained with 802.11ac than 802.11n. Data rates of 802.11ac dropped significantly at further distances when compared to 802.11n. The fluctuation in performance of 802.11ac could be due to the deployment of other APs within the R.F. environment. As 802.11 ac requires an $80 \mathrm{MHz}$ channel to achieve maximum performance, made up of two $40 \mathrm{MHz}$ channels, both channels have to be available in order to transmit maximum data rate over the $80 \mathrm{MHz}$ channel. APs within the environment may have been operating over one $40 \mathrm{MHz}$ channel instead. The limiting of 802.11ac to one 40 $\mathrm{MHz}$ channel had a significant impact on performance and is likely to be the explanation for the poor performance. However, as the experiment replicates a typical office environment, there are likely to be other APs operating in the wireless environment in practice, hence the scenario is realistic.

Packet loss was significantly higher over 802.11ac than $802.11 n$ with the highest percentage of packet loss recorded at the nearest distance $(0.097 \%)$. This could also support the theory of interference with 802.11ac due to other 802.11n APs within the environment. Furthermore, 802.11n continued to present consistency with minimal packet loss at the closest distance $(0.002 \%)$ which as expected, increased with distance.

Although 802.11ac achieved some of the highest data rates recorded throughout the experiment, it rarely showed consistency, performing on average at $21.7 \%$ of its full potential. 802.11ac also had significantly higher packet loss than $802.11 \mathrm{n}$ with longer distances and obstacles having a serious impact on 802.ac performance. On average, 802.11n performed better that of $802.11 a c$, achieving $44.9 \%$ of its full potential with a consistently lower error rate.

Both standards did not achieve their full potential when averaged. However, 802.11ac was more volatile and susceptible to distance, obstacles and interference. It did not present a significant increase to performance over $802.11 \mathrm{n}$ within an office environment over distances. The 802.11ac specification however, suggests otherwise.

\section{CONCLUSIONS AND FURTHER WORK}

The review showed that work by Dianu et al. [3] explored the performance of 802.11ac in an indoor environment. Bejarano and Knightly [2] covered channelization and MIMO issues. Gauntlett [11] provided an insight to Modulation and Coding Scheme Values. Several software programs were evaluated to determine good test cases. Wireshark and WirelessMon were chosen for monitoring purposes. An office environment like experimental scenario was designed and used to collect data about the practical capability of 802.11ac. It was found that there are a vast range of parameter settings configurable within the Cisco wireless controller. In order to carry out each test case in a feasible time, variables were chosen that optimised data rate. The laboratory used to carry out the experiment was limited in size to fifteen meters. It would have been better to perform the experiment in a laboratory of twenty five meters to avoid transmitting through the wall, which had a noticeable impact on data rate. Ideally, the project should have been repeated in an RF anechoic chamber. 
Graphs containing data rates for each standard, at each distance, over each channel have been analysed to compare to the highest, lowest and the mean data rates. Additionally more detailed graphs could be created from screen shots of data taken at each distance showing the number of interfering APs present at each distance. The AP implemented for the experiment was a Wave 1 Cisco Aironet 2702i. Ideally, the experiment would have benefited from a Wave 2 Cisco Aironet AP such as the Cisco Aironet 2800i. This would be able to demonstrate a greater 802.11ac performance with theoretical data rates of up to 2.6Gb/s rather than Wave 1's $866.7 \mathrm{Mb} / \mathrm{s}$. Another improvement would be to test the impact of interference from 802.11n signals on 802.11ac when transmitting simultaneously. The findings showed inconsistencies with 802.11ac which were may be caused $802.11 n$ transmissions. Whereas $802.11 n$ proved to be consistent, providing evidence that there was likely to be little interference. By implementing an additional AP that performs 802.11n transmission alongside 802.11ac, the experiment would then provide data about signals interfering with 802.11ac performance.

\section{REFERENCES}

[1] C. Zhuo and S. Hajime, "Performance of 802.11n WLAN with transmit antenna selection in measured indoor channels," in Communications Theory Workshop, 2008. AusCTW 2008. Australian, 2008, pp. 139-143.

[2] O. Bejarano and E. W. Knightly, "Virtual MISO triggers in Wi-Fi-like networks," 2013 Proceedings IEEE INFOCOM, p. 1582, 2013.

[3] M. D. Dianu, J. Riihij, x00E, rvi, and M. Petrova, "Measurement-based study of the performance of IEEE 802.11ac in an indoor environment," in 2014 IEEE International Conference on Communications (ICC), 2014, pp. 5771-5776.

[4] M. Kassner. (2007, March). What is the big deal about 802.11n? Available:

http://www.techrepublic.com/blog/mobile-enterprise/what-isthe-big-deal-about-80211n/

[5] Santumon.S.D and B. R. Sujatha, "Space-Time Block Coding (STBC) for Wireless Networks," International Journal of Distributed and Parallel Systems, p. 183, 2012.

[6] F. Riera-Palou and G. Femenias, "Improving STBC Performance in IEEE 802.11n Using Group-Orthogonal Frequency Diversity," 2008 IEEE Wireless Communications \& Networking Conference, p. 193, 2008.
[7] M. Gast, 802.11ac : a survival guide / Matthew Gast: Sebastopol, CA : O'Reilly, 2013., 2013.

[8] D. E. Capano, "Understanding modulation and coding schemes," Control Engineering, vol. 61, pp. 26-26, 2014.

[9] G. Combs, "Wireshark. 2.0.2," ed, 2016.

[10]N. Soft, "AdapterWatch. 1.05," ed, 2009.

[11]D. Gauntlett. (2012, March). Demystifying Modulation and Coding Scheme (MCS) Index Values. Digital Air Wireless. Available: http://www.digitalairwireless.com/wirelessblog/recent/demystifying-modulation-and-coding-schemeindex-values.html 